\title{
Demografie und Einkommensungleichheit
}

Deutschland steht vor einem gewaltigen demografischen Umbruch. Die Alterung (und Schrumpfung) der bundesdeutschen Bevölkerung dürfte auch spürbare Auswirkungen auf die personelle Einkommensverteilung haben. Es werden voraussichtlich nicht nur die Verteilungskämpfe in den bundesdeutschen Sicherungssystemen an Schärfe gewinnen. Vielmehr dürften sich aktuellen Trends zufolge auch die erwarteten wirtschafts- und haushaltsstrukturellen Veränderungen wesentlich auf die gemessene Einkommensungleichheit in Richtung Ungleichheitsanstieg auswirken. ${ }^{1}$

\section{Einleitung}

Der Beitrag setzt sich mit den Zusammenhängen zwischen den Größen Demografie einerseits und Einkommensverteilung bzw. -ungleichheit andererseits auseinander. Vor dem Hintergrund des bundesdeutschen Alterungsprozesses wird das gesellschaftliche Verhältnis zwischen den Generationen - zugespitzt: zwischen den "Alten“ und den "Jungen“ - zunehmend bedeutsamer. Unter der begründeten Prämisse, dass individuelle Entfaltungsmöglichkeiten sich zuallererst aus wirtschaftlichen Gestaltungsspielräumen ergeben, erscheint in diesem Zusammenhang eine korrespondierende Wohlstandsanalyse angebracht. Insbesondere aufgrund datenbezogener Restriktionen bezieht sich die vorliegende Arbeit durchgängig auf den Wohlstandsindikator Einkommen, genauer gesagt: auf das Haushaltsnettoäquivalenzeinkommen.

Die Alterung einer Gesellschaft betrifft primär indirekte Demografieeffekte, welche nicht zuletzt über das SteuerTransfer-System vermittelt werden. Hier stehen in Deutschland insbesondere das Umlageverfahren der gesetzlichen Rentenversicherung und die hiermit verbundene intergenerative Verteilungsproblematik im Mittelpunkt. Auch beeinflusst die demografische Entwicklung sowohl die Einkommensentstehung (über Änderungen in den relativen Faktor- wie Güterpreisen beziehungsweise über hiermit gekoppelte negative Anreize bezüglich einer etwaigen Arbeitsaufnahme) als auch die Einkommensverwendung (über altersspezifische Konsum- und Sparquoten oder über altersdifferenzierte Konsumstrukturen), wobei die Aspekte der Einkommensverteilung, der Einkommensentstehung und der Ein- kommensverwendung in interdependenten Zusammenhängen zueinander stehen.

Da sich gesellschaftliche Konflikte unmittelbar auf der Querschnittsebene offenbaren, ist die Betrachtung der querschnittlichen Einkommensungleichheit von beträchtlichem sozialpolitischen Interesse - nicht zuletzt für die praktische Sozialpolitik.

Paglin (1975) und Kuznets (1976) eröffneten Mitte der 1970er Jahre eine international geführte wissenschaftliche Debatte darüber, wie demografische Größen auf die gemessene (Querschnitts-)Einkommensungleichheit einwirken. Ihnen zufolge ist von einer positiven statistischen Korrelation zwischen Haushaltsgröße und -einkommen sowie von einem umgekehrt u-förmigen Verlauf der Haushaltseinkommen in Abhängigkeit vom Alter des Haushaltsvorstandes auszugehen. Beide Beziehungen zusammengenommen, formulieren Abhängigkeiten der Haushaltseinkommensverteilung von der Verteilung der Haushalte nach ihrer Größe und von der Altersverteilung (in diesem Fall: von der Altersverteilung der Haushaltsvorstände). Neben der Altersverteilung sind aber auch die durch den sozialen Wandel ausgelösten Veränderungen in den Haushaltsstrukturen besonders ungleichheitsrelevant. Beispielhaft zu nennen ist hier eine wachsende Bildungs- und Einkommenshomogamie ${ }^{2}$ beim Heiratsverhalten beziehungsweise bei der Haushaltsformation, wie sie etwa Western et al. (2008) für die jüngere Vergangenheit in den USA festgestellt haben.

Abschnitt 2 nimmt die Rolle der Demografie bei der Erklärung der gemessenen Einkommensungleichheit in den Blick, indem grundlegende Wirkmechanismen und Übertragungskanäle zwischen Demografie und Einkommensungleichheit thematisiert werden. Auf diesen Überlegungen aufbauend, leistet Abschnitt 3 ei- nen eigenen empirischen Beitrag zur Debatte. Schließlich werden in Abschnitt 4 die Überlegungen und Befunde des Beitrags zusammengefasst und weitere Forschungsbedarfe skizziert.

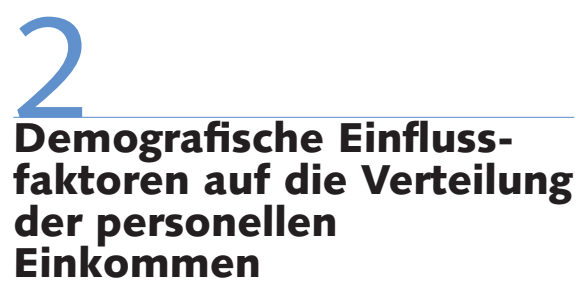

Im Folgenden werden Grundzusammenhänge zwischen Demografie und Einkommensungleichheit erörtert, wobei von einem Wirkungszusammenhang von der Demografie hin zur Einkommensungleichheit ausgegangen wird. Von den gegenteiligen Einflüssen - d.h. von der Einkommensungleichheit hin zur Demografie - wird hingegen abstrahiert.

Hinsichtlich des Zusammenhangs zwischen Demografie und Einkommensungleichheit ist zwischen direkten und indirekten Demografieeffekten zu unterscheiden. Während die reine Fixierung der demografischen Struktur eines Basisjahres

1 Die Daten dieser Publikation beruhen auf Zahlen des Sozioökonomischen Panels (SOEP) am Deutschen Institut für Wirtschaftsforschung (DIW), Berlin. Die SOEP-Welle 2009 stand dem Autor zum Zeitpunkt der Ausarbeitung dieses Artikels noch nicht zur Verfügung. Der Autor dankt den zwei anonymen Gutachtern für deren wertvolle Hinweise.

2 Homogamie bezeichnet die Ähnlichkeit bzw. Gleichheit von Partnern.

Jürgen Faik, Dr., ist Diplom-Volkswirt bei FaMa-Neue Frankfurter Sozialforschung in Frankfurt am Main. Arbeitsschwerpunkte: Soziale Ungleichheit, Arbeitsmarkttheorie und -politik, Empirische Sozialforschung. e-mail: faik@fama-nfs.de 
in Verteilungsanalysen nur die direkten Demografieeffekte erfasst, zielen die indirekten demografischen Effekte auf die Veränderung der verteilungsrelevanten ökonomischen Prozesse ab. Zu den letztgenannten ökonomischen Prozessen gehören neben individuellen Anpassungsreaktionen (etwa auf dem Arbeitsmarkt mit Abhängigkeiten vom eigenen, aber auch vom Alter der anderen Marktteilnehmer) auch fiskalische Aspekte wie die Einhaltung von (intra- und intertemporalen) Budgetrestriktionen des Staates beziehungsweise der sozialen Sicherungssysteme (vgl. von Weizsäcker 1994, S. 33f.).

Vereinfacht kann man die nachfolgenden Wirkmechanismen von der Demografie hin zur (Äquivalenz-)Einkommensungleichheit unterscheiden (vgl. in diesem Zusammenhang auch Heerink 1994, S. 2): Gemäß Abbildung 1 und unter Bezugnahme auf die unterschiedlichen Einkommensquellen - Arbeitseinkommen, Kapitaleinkommen und (Netto-)Transfereinkommen - existieren mit dem Arbeitsmarkt, dem Kapitalmarkt und dem Steuer-TransferSystem drei wesentliche Übertragungskanäle von Demografie-Änderungen auf die (Äquivalenz-)Einkommensungleichheit. Zusätzlich sind noch die Einflusskanäle „Einkommensverwendung“ und „Makroökonomische Ebene" in Abbildung 1 dargestellt, denen allerdings im Vergleich zu den drei erstgenannten Kanälen eine nachgeordnete Rolle zukommt und die zum Teil von den drei Hauptkanälen abhängig sind (was allerdings in Abbildung 1 aus Vereinfachungsgründen ebenso wenig wie die Interdependenzen zwischen den einzelnen Übertragungskanälen dargestellt wird).

\subsection{DIREKTE DEMOGRAFIEEINFLÜSSE}

Als direkte Demografieeinflüsse auf die Ungleichheit der Haushaltsnettoäquivalenzeinkommen sind die Effekte zu nennen, welche von der Fertilität, der Mortalität, der Migration und von der Haushaltsstruktur ausgehen. Das Haushaltsnettoäquivalenzeinkommen wird dadurch ermittelt, dass das jeweilige Haushaltsnettoeinkommen durch einen haushaltsbezogenen Äquivalenzskalenwert dividiert wird. Der haushaltsbezogene Äquivalenzskalenwert ist die Summe individueller Bedarfsgewichte für die einzelnen Haushaltsmitglieder. Das entsprechend ermittelte Haushaltsnettoäquivalenzeinkommen wird in einem weiteren analytischen Schritt (fik-

\section{Abb. 1: Idealisierte Einflusskanäle der Demografie auf die Einkommensungleichheit}

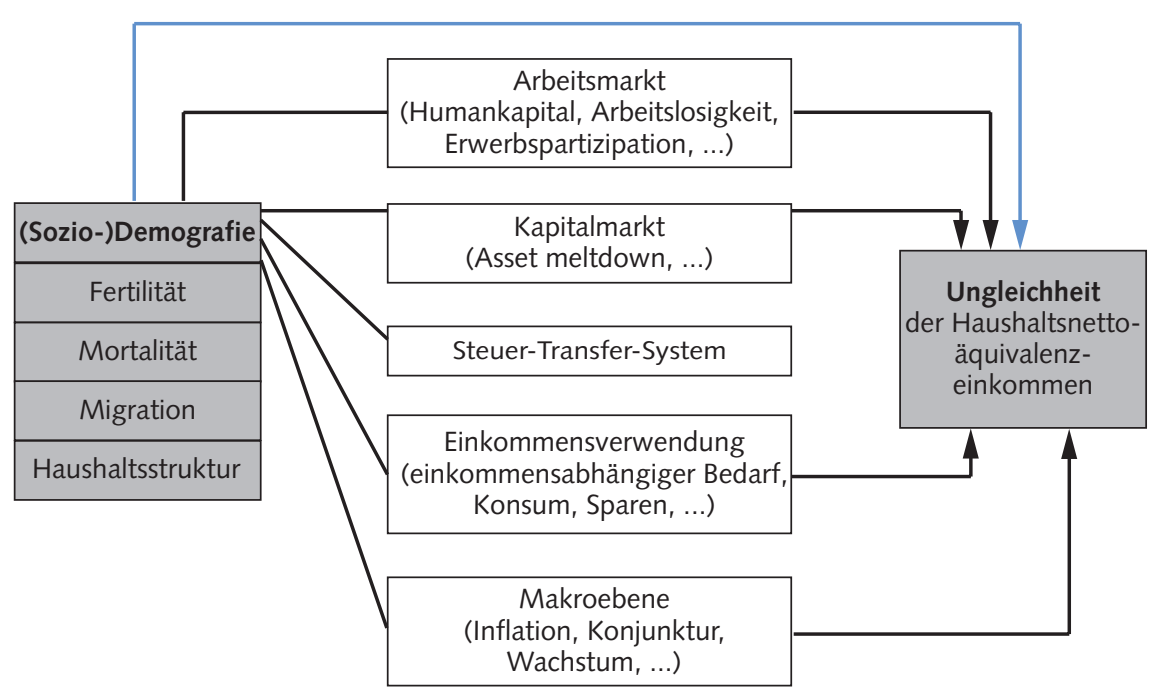

\footnotetext{
$\longrightarrow$ Bevölkerungsgröße, -anteile

$\longrightarrow$ (Äquivalenz-)Einkommen,

(Äquivalenz-)Einkommensanteile
}

Quelle: Darstellung des Autors.

WSI MITTELUNGEN

tiv) jedem betreffenden Haushaltsmitglied zugeordnet, um den Wohlstand des Letztempfängers von Wohlstand, nämlich des Individuums, messen zu können.

Hinsichtlich der haushaltsstrukturellen Einflüsse auf die personelle (Äquivalenz-) Einkommensungleichheit sind insbesondere zwei Aspekte relevant. Erstens sind Wohlfahrtsgewinne zu nennen, welche sich über Größenvorteile im Haushaltskontext ergeben. Zweitens kann die $\mathrm{Zu}$ sammenführung kleinerer zu größeren Haushaltsgemeinschaften dazu führen, dass bis dato wohlstandsbezogen relativ schlecht situierte Personen an dem vergleichsweise hohen Einkommen eines "neuen“ Haushaltsmitgliedes partizipieren. Bei nicht allzu ausgeprägter positiver Korrelation der betreffenden Individualeinkommen ist die gemessene ökonomische Ungleichheit dann geringer, wenn Erwachsene ihre Einkommen in Paarbeziehungen miteinander koppeln, als wenn sie unabhängig voneinander allein leben. Wenn demgemäß im Umkehrschluss der Anteil von Paaren sinkt und die positive Korrelation zwischen den Partnereinkommen (im Sinne von Homogamie) steigt, erhöht sich (tendenziell) die Ungleichheit. Ähnliches gilt, wenn Niedrigeinkommensbezieher überproportional allein lebend (oder allein erziehend) sind (vgl. Burtless 2009, S. 437ff.).
Auf die Effekte der Fertilität, der Mortalität und der Migration auf die Äquivalenzeinkommensungleichheit, welche im Übrigen nicht allgemeingültig anzugeben sind (vgl. in diesem Kontext etwa die Betrachtungen in Pohmer 1989 beziehungsweise für einen entsprechenden Überblick Faik 2010b, S. 13ff.), wird in diesem Beitrag deshalb nicht näher eingegangen, weil sie eher für Längsschnitt- als für die hier dargelegten Querschnittsbetrachtungen relevant erscheinen.

\subsection{INDIREKTE DEMOGRAFIE- EINFLÜSSE}

Wie oben erwähnt, sind hinsichtlich der indirekten Demografieeinflüsse auf die personelle Einkommensverteilung primär die Effekte von Bedeutung, welche über die Kanäle Arbeitsmarkt, Kapitalmarkt und Steuer-Transfer-System vermittelt werden.

Hinsichtlich des Einflusskanals Arbeitsmarkt kann auf demografisch gegliederte Arbeitseinkommensdifferenzen, d.h. auf Alters-ArbeitseinkommensProfile, Bezug genommen werden, welche humankapitaltheoretisch begründet werden können. So kann argumentiert werden, dass typischerweise die meisten Investitionen in Humankapital (Ausbildung, On-the-job-Training usw.) in jün- 
geren Lebensjahren stattfinden. Hiermit gehen - als Folge der Investitionskosten - Arbeitseinkommensverminderungen in jungen Lebensjahren einher, während die Erträge derartiger Investitionen erst in späteren Lebensjahren wirksam werden. Hieraus folgt ein positiver Zusammenhang zwischen Alter und (Arbeits-) Einkommen. Eine Erhöhung von $\mathrm{Hu}-$ mankapitalinvestitionen führt zu einem steileren Alters-Arbeitseinkommens-Profil. In späteren Lebensjahren müssen den skizzierten Anstiegen die Abschreibungen als Folge körperlicher und geistiger „Abnutzungen " gegenübergestellt werden, sodass sich die Steigung des Alters-Einkommens-Profils in den korrespondierenden Altersbereichen vermindert (vgl. Becker 1964; Mincer 1974; auch Heerink 1994, S. 178f.).

Die vorstehenden Betrachtungen bezogen sich auf Alters-ArbeitseinkommensProfile im Längsschnitt. Man kann AltersArbeitseinkommens-Profile aber auch im (in dieser Arbeit relevanten) Querschnitt betrachten, indem man auf die durchschnittlichen Arbeitseinkommen verschiedener Altersgruppen zu einem bestimmten Zeitpunkt abstellt - im Unterschied zur Längsschnittperspektive, bei der für verschiedene Kohorten die Arbeitseinkommensentwicklung im Zeitablauf analysiert wird. Wegen konjunktureller, institutioneller und ähnlicher Trends bezüglich der Arbeitseinkommensniveaus beziehungsweise bezüglich der diese Niveaus determinierenden Faktoren unterscheiden sich Alters-Arbeitseinkommens-Profile im Querschnitt typischerweise von solchen im Längsschnitt. Querschnittliche AltersArbeitseinkommens-Profile weisen üblicherweise einen umgekehrt u-förmigen Verlauf - über die einzelnen Altersklassen hinweg - auf.

Ein nennenswerter Aspekt, der Demografie und Kapitalmärkte verbindet, besteht darin, dass eine verstärkte Inanspruchnahme der Kapitalmärkte - etwa als Folge institutioneller Teilumstiege zum Beispiel der Alterssicherung auf das Kapitaldeckungs- anstelle des Umlageverfahrens - dazu führen kann, dass zu einem bestimmten Zeitpunkt eine vergrößerte Zahl von Wertpapierverkäufern einer verringerten Zahl potenzieller Käufer gegenüberstehen kann. Dies könnte sich unmittelbar preis- und somit renditesenkend auswirken (sogenannte „Asset-meltdownThese"), was tendenziell zu einer gleichmä- ßigeren Verteilung der Kapitaleinkommen führen dürfte.

Ein weiterer Zusammenhang zwischen Demografie und Kapitalmarktgeschehen ergibt sich aus der Lebenszyklus-Hypothese, die auf Ando/Modigliani (1963) zurückgeht, für Deutschland allerdings nur eingeschränkt Gültigkeit beanspruchen kann (vgl. hierzu etwa Börsch-Supan/ Essig 2002). Gemäß der LebenszyklusHypothese optimiert ein Individuum beziehungsweise ein privater Haushalt jederzeit seinen (Güter- und Freizeit-)Konsum über seine gesamte Lebenszeit hinweg. Auf diese Art und Weise wird sein Lebensgesamtnutzen maximiert. Gemäß der Lebenszyklus-Hypothese glätten die privaten Haushalte ihren Konsum durch Ersparnisvariation, konkret: durch Entsparen in der Ausbildungs- und Rentenphase sowie durch Sparen in der Erwerbsphase. Ihre Vermögensbestände wachsen bis zur Rentenphase, ehe sie danach schrittweise abgebaut werden. In einer alternden Gesellschaft bewirkt das Entsparen im Alter, d.h. der Vermögensabbau im Alter, über den dadurch induzierten Rückgang der Vermögenseinkommen der Älteren tendenziell eine Reduktion der Vermögenseinkommensungleichheit und damit ceteris paribus auch der gesamten Einkommensungleichheit. So werden - durchaus unter Berücksichtigung des Hinterlassens eines Erbes - die überdurchschnittlich hohen Vermögenseinkommen der Älteren zunehmend in Richtung der niedrigeren Vermögenseinkommen der jüngeren Altersgruppen reduziert.

Um den Einflusskanal Steuer-TransferSystem zu illustrieren, kann man analog zu von Weizsäcker (1994, S. 35ff.) in einem komparativ-statischen Gleichgewichtsmodell aus Vereinfachungsgründen von lediglich zwei Altersgruppen ausgehen: von Erwerbstätigen und von Altersrentnern. Für beide Gruppen können Einkommensgleichungen (im Sinne des jeweiligen Nettogesamteinkommens) formuliert werden, und unter Bezugnahme auf die ersten beiden Momente der Verteilung - arithmetischer Mittelwert und Varianz - ergeben sich dann die betreffenden Gesamtwerte aus den gruppenspezifischen Mittelwerten und Varianzen. Als Ungleichheitsmaß verwendet im Übrigen von Weizsäcker folgerichtig den quadrierten Variationskoeffizienten, d.h. das Verhältnis aus Einkommensvarianz und quadriertem (arithmetischen) Einkommensmittel- wert. Durch partielle Ableitungen dieses Ungleichheitsmaßes nach den relevanten Einflussgrößen können die jeweiligen Ungleichheitseinflüsse hergeleitet werden. Im Rahmen einer Budgetinzidenzanalyse können dabei budgetäre Restriktionen für das Steuer- und Transfersystem explizit berücksichtigt werden.

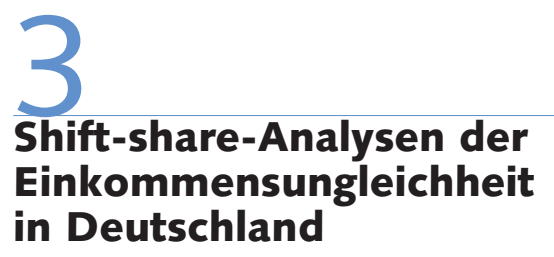

In der Literatur finden sich - im internationalen Maßstab - zahlreiche komparativstatische Inzidenzanalysen für den Einfluss der Demografie auf die Einkommensungleichheit (zu einem entsprechenden Überblick siehe Burtless 2009 oder Faik 2010b, S. 32ff.). In solchen Analysen wird typischerweise die Bevölkerungsstruktur eines bestimmten Basisjahres über den jeweiligen Betrachtungszeitraum hinweg konstant gehalten, und es wird aus den Unterschieden zu den Ungleichheitswerten bei Zugrundelegung der tatsächlichen Bevölkerungsanteile auf den demografischen Einkommensungleichheitseinfluss geschlossen. Diese vereinfachende, sogenannte "Shift-share-Vorgehensweise“ impliziert, dass Veränderungen in der Bevölkerungsstruktur nicht das Ausmaß der Ungleichheit innerhalb der Subgruppen und auch nicht die Unterschiede in den Durchschnittseinkommen zwischen den Subpopulationen beeinflussen. Es werden damit nur die direkten Demografieeffekte auf die gesamte Einkommensungleichheit berücksichtigt.

Alternativ - gegebenenfalls additiv - lassen sich in Shift-share-Analysen aber auch die ökonomischen Parameter konstant halten. In Ceteris-paribus-Vergleichen mit den tatsächlichen Ungleichheitswerten können dann die Effekte von Änderungen in den ökonomischen Bedingungen auf die personelle Einkommensungleichheit komparativ-statisch untersucht werden.

Um entsprechende Einflüsse auf die gemessene (Äquivalenz-)Einkommensungleichheit zu erfassen, erscheint es naheliegend, einen - etwa nach verschiedenen Altersgruppen - dekomponierbaren Ungleichheitsindikator zu verwenden. Ein solcher Indikator ist ein normierter Varia- 
tionskoeffizient (CV), der als die Hälfte des quadrierten Variationskoeffizienten definiert ist, wobei der Variationskoeffizient - wie oben angedeutet - das Verhältnis der Standardabweichung zum arithmetischen Einkommensmittelwert angibt. Man kann $\mathrm{CV}$ in eine Intra- und eine Intergruppenkomponente zerlegen (Übersicht 1). Hierbei wird die Intragruppenkomponente geprägt durch die Bevölkerungs- und die Einkommensanteile der einzelnen Gruppen und durch die gruppeninternen $\mathrm{CV}$ Ungleichheitswerte. Die Intergruppenkomponente hat die Bevölkerungs- und die Einkommensanteile der diversen Gruppen als Elemente. ${ }^{3}$

\subsection{SHIFT-SHARE-ANALYSEN DER EINKOMMENSUNGLEICHHEIT IN DEUTSCHLAND 1995-2007}

Bezugnehmend auf die vorstehend angesprochene Zerlegung des genannten normierten Variationskoeffizienten, bieten sich drei kontrafaktische Situationen im Rahmen eigener Shift-share-Analysen an:

- Konstante Bevölkerungsanteile der voneinander unterschiedenen soziodemografischen Gruppen $\mathrm{w}_{\mathrm{g}}$,

- konstante Äquivalenzeinkommens-Mittelwerte $\mu$ beziehungsweise $\mu_{\mathrm{g}}$ und

- konstante gruppeninterne Ungleichheitswerte $\mathrm{CV}_{\mathrm{g}}$.

Diese Shift-share-Betrachtungen beziehen sich durchgängig auf das in Abschnitt 2 näher erläuterte Konzept des Haushaltsnettoäquivalenzeinkommens (im Übrigen inklusive eines fiktiven Mietwertes für selbst genutztes Wohneigentum). Konkret werden in dieser Arbeit die Bedarfsgewichte der sogenannten „neuen OECD-Skala"verwendet; d. h.: 1,0 für den Haushaltsvorstand sowie 0,5 für weitere Haushaltsmitglieder ab 15 Lebensjahren und 0,3 für weitere Haushaltsmitglieder bis 15 Lebensjahre. ${ }^{4}$

Als Datenbasis liegt den nachfolgend präsentierten Ergebnissen das Sozioökonomische Panel (SOEP) in seiner CNEFVariante zugrunde. ${ }^{5}$ Der Untersuchungszeitraum erstreckt sich von 1995 bis 2007 (für Gesamtdeutschland), wobei als Bezugsjahr für die einzelnen Shift-shareBetrachtungen jeweils das Ausgangsjahr 1995 fungiert. Es wird deshalb auf das Jahr 1995 Bezug genommen, weil zu diesem Zeitpunkt der Transformationsprozess in

\section{Übersicht 1: Formale Darstellung des normierten Variations- koeffizienten (CV) der personellen Ungleichheitsverteilung}

Zerlegung des CV in eine Inter- und Intragruppenkomponente

$C V=\sum_{g=1}^{G} v_{g}^{2} \cdot w_{g}^{-1} \cdot C V_{g}+\frac{1}{2} \cdot\left\{\left[\sum_{g=1}^{G} w_{g} \cdot\left(\frac{\mu_{g}}{\mu}\right)^{2}\right]-1\right\}$

Fiktiver CV in kontrafaktischen Situationen

Konstante Bevölkerungsanteile

$C V_{t}=\sum_{g=1}^{G} v_{g, t}^{2} \cdot w_{g, 1995}^{-1} \cdot C V_{g, t}+\frac{1}{2} \cdot\left\{\left[\sum_{g=1}^{G} w_{g, 1995} \cdot\left(\frac{\mu_{g, t}}{\mu_{t}}\right)^{2}\right]-1\right\}$

mit: $t=1995,1996, \ldots, 2007$

Konstante Mittelwerte der Äquivalenzeinkommen

$C V_{t}=\sum_{g=1}^{G} v_{g, 1995}^{2} \cdot w_{g, t}^{-1} \cdot C V_{g, t}+\frac{1}{2} \cdot\left\{\left[\sum_{g=1}^{G} w_{g, t} \cdot\left(\frac{\mu_{g, 1995}}{\mu_{1995}}\right)^{2}\right]-1\right\}$

mit: $t=1995,1996, \ldots, 2007$.

Konstante gruppeninterne Ungleichheitswerte

$C V_{t}=\sum_{g=1}^{G} v_{g, t}^{2} \cdot w_{g, t}^{-1} \cdot C V_{g, 1995}+\frac{1}{2} \cdot\left\{\left[\sum_{g=1}^{G} w_{g, t} \cdot\left(\frac{\mu_{g, t}}{\mu_{t}}\right)^{2}\right]-1\right\}$

mit: $\mathrm{t}=1995,1996, \ldots, 2007$

Anmerkung: $\mathrm{CV}=$ normierter Variationskoeffizient, $\mathrm{CV}_{\mathrm{g}}=$ normierter Variationskoeffizient innerhalb der Gruppe $g, \mu_{\mathrm{g}}=$ arithmetischer Einkommensmittelwert der Gruppe $g_{r} \mu=$ allgemeiner Einkommensmittelwert. Die Gewichtungsfaktoren $w_{g}$ $\left(=\mathrm{n}_{\mathrm{g}} / \mathrm{n}\right)$ repräsentieren die Bevölkerungsanteile der verschiedenen Personengruppen $\mathrm{g}(\mathrm{g}=1,2, \ldots, \mathrm{G}), \mathrm{und}_{\mathrm{g}}\left(=\mathrm{w}_{\mathrm{g}} \mu_{\mathrm{g}} / \mu\right)^{8}$ bezeichnet den gruppenspezifischen Anteil am aggregierten Einkommen; $\mathrm{n}=$ allgemeine Bevölkerungsgröße, $\mathrm{n}_{\mathrm{g}}=$ Anzahl der ruppe $g$.

Quelle: Darstellung des Autors.

WSI MITTEILUNGEN

Ostdeutschland weitgehend zum Erliegen gekommen war; die Wahl eines früheren Basisjahres wäre demgegenüber durch die Sondereinflüsse des ostdeutschen Transformationsprozesses in vermutlich höherem Maße geprägt gewesen. ${ }^{6}$

Es werden drei Altersgruppen voneinander unterschieden: bis 29 Jahre, 30-59 Jahre sowie 60 Jahre und älter. Diese Dreiteilung lehnt sich an Hauser (2007) an, dem zufolge eine Generation etwa 30 Lebensjahre umfasst. In diesem (pragmatischen) Sinne wird zu einem gegebenen Zeitpunkt von drei gleichzeitig lebenden Generationen ausgegangen, sozusagen von der Großeltern-, der Eltern- und der Kindergeneration. Auf diese Weise kann der Frage nach intergenerativen Verteilungsunterschieden prononciert nachgegangen werden. Weil in diesem Artikel auf das individuelle Wohlstandsniveau abgestellt wird, wird einer Person jeweils das Haushaltsnettoäquivalenzeinkommen des Haushaltes zugeordnet, dem sie angehört.

In Tabelle 1 sind die entsprechenden Sensitivitätsergebnisse angegeben. Die folgenden Abschnitte bauen auf diesen Ergebnissen auf. ${ }^{7}$
3 Zu den (Zerlegungs-) Eigenschaften von CV bzw. anderer Ungleichheitsindikatoren aus der gleichen Klasse der Generalized-Entropy-Indikatoren siehe Faik 2010a, S. 8ff. und die dortigen Literaturhinweise. Eigene Alternativberechnungen bezogen sich auf die mittlere logarithmische Abweichung, welche ebenfalls zur Klasse der Generalized-Entropy-Indikatoren zählt. Sie reagiert im Vergleich zu CV stärker auf Verteilungsänderungen im unteren bzw. mittleren (Äquivalenz-) Einkommensbereich. Diese Alternativberechnungen bestätigten zwar nicht durchgängig, aber doch im Wesentlichen (d. h insbesondere im Hinblick auf die von den prozentualen Abweichungen her bedeutsamste Variante konstanter gruppeninterner Ungleichheitswerte) die folgenden auf CV-Basis ermittelten Befunde.

4 Alternative Berechnungen des Autors auf Basis der „alten OECD-Skala“ (Haushaltsvorstand: 1,0; weitere Haushaltsmitglieder ab 15 Lebensjahren: 0,7; weitere Haushaltsmitglieder bis 15 Lebensjahre: 0,5) erbrachten zwar etwas höhere CV-Ungleichheitswerte, aber keine größeren qualitativen Ergebnisunterschiede.

5 CNEF steht für Cross-National Equivalent File. Eine umfassende Beschreibung der SOEP/CNEFVariante findet sich in Burkhauser et al. 2001.

6 Gleichwohl haben eigene Alternativberechnungen mit dem Jahr 1992 als Basisjahr die grundsätzlichen empirischen Aussagen dieses Artikels auf der qualitativen Ebene bestätigt.

$7 \quad$ Wie eigene Bootstrapping-Analysen (mit jeweils 1.000 Stichprobenziehungen) zeigten, sind die in Tabelle 1 ausgewiesenen Ungleichheitsstrukturen - bei Zugrundelegung von 95-\%-Konfidenzintervallen und ohne Hochrechnungsfaktoren - statistisch als robust zu bezeichnen. 
Tabelle 1: Normierte Variationskoeffizienten (CVS) der personellen Haushaltsnettoäquivalenzeinkommen in Deutschland, Shift-share-Szenarien

\begin{tabular}{|c|c|c|c|c|}
\hline Jahr & Tatsächlicher CV (a) & $\begin{array}{l}\text { Fiktiver CV bei konstanter } \\
\text { Bevölkerungsstruktur (b) }\end{array}$ & $\begin{array}{l}\text { Fiktiver CV bei konstanten } \\
\text { Einkommensanteilen (c) }\end{array}$ & $\begin{array}{l}\text { Fiktiver CV bei konstanten } \\
\text { gruppenspezifischen CVs (d) }\end{array}$ \\
\hline 1995 & 0,1589 & $0,1589(0,0)$ & $0,1589(0,0)$ & $0,1589(0,0)$ \\
\hline 1996 & 0,1357 & $0,1334(-1,7)$ & $0,1369(+0,9)$ & $0,1602(+18,0)$ \\
\hline 1997 & 0,1512 & $0,1481(-2,1)$ & $0,1533(+1,4)$ & $0,1601(+5,9)$ \\
\hline 1998 & 0,1467 & $0,1425(-2,9)$ & $0,1507(+2,7)$ & $0,1594(+8,6)$ \\
\hline 1999 & 0,1684 & $0,1612(-4,3)$ & $0,1708(+1,5)$ & $0,1601(-4,9)$ \\
\hline 2000 & 0,1503 & $0,1460(-2,9)$ & $0,1534(+2,0)$ & $0,1601(+6,5)$ \\
\hline 2001 & 0,2409 & $0,2354(-2,3)$ & $0,2398(-0,4)$ & $0,1590(-34,0)$ \\
\hline 2002 & 0,2146 & $0,2075(-3,3)$ & $0,2133(-0,6)$ & $0,1602(-25,4)$ \\
\hline 2003 & 0,2823 & $0,2733(-3,2)$ & $0,2808(-0,5)$ & $0,1607(-43,1)$ \\
\hline 2004 & 0,2266 & $0,2171(-4,2)$ & $0,2239(-1,2)$ & $0,1582(-30,2)$ \\
\hline 2005 & 0,3049 & $0,2961(-2,9)$ & $0,3046(-0,1)$ & $0,1608(-47,3)$ \\
\hline 2006 & 0,3178 & $0,3031(-4,6)$ & $0,3117(-1,9)$ & $0,1591(-49,9)$ \\
\hline 2007 & 0,4037 & $0,3891(-3,6)$ & $0,4020(-0,4)$ & $0,1587(-60,7)$ \\
\hline
\end{tabular}

\section{KONSTANTE BEVÖLKERUNGSANTEILE}

Die erste kontrafaktische Situation ist wie oben dargelegt - dadurch charakterisiert, dass die Bevölkerungsanteile der drei voneinander unterschiedenen Altersgruppen konstant gehalten werden, und zwar auf dem Niveau der (Populations-) Werte des Ausgangsjahres 1995 (Übersicht 1).

Empirisch zeigt sich in diesem Kontext, dass die durch diesen Vergleich zwischen tatsächlicher und kontrafaktischer Situation ermittelten direkten Effekte der demografischen Veränderungen ungleichheitserhöhend gewirkt haben. Bis 1999 ergab sich hierbei insofern eine gewisse Dynamik, als die entsprechenden CV-Ungleichheitserhöhungen prozentual stetig wuchsen; für die Zeit ab 2000 wird ein zyklisches Muster evident (auf dem Niveau von Ungleichheitserhöhungen zwischen ca. 2,3 und ca. 4,6\%; Tabelle 1, Spalte $b$ ).

Die einzelnen Ungleichheitserhöhungen sind im Rahmen der betreffenden Shift-share-Analyse die Folge der im Untersuchungszeitraum festgestellten Alterung der bundesdeutschen Bevölkerung. Für den hier relevanten Zeitraum ist (auf SOEP-Grundlage) in Gesamtdeutschland der Anteil der unter 30-Jährigen von ca. $36 \%$ auf gut $31 \%$ gesunken und jener der 60-Jährigen und Älteren von etwa $23 \%$ auf ca. $27 \%$ gestiegen. Dies zeigt den bereits heute feststellbaren Bedeutungsgewinn der Älteren hinsichtlich der jeweiligen Populationsanteile in Deutschland auf.

\section{KONSTANTE ÄQUIVALENZ- EINKOMMENSMITTELWERTE}

Im Rahmen der in diesem Abschnitt relevanten Shift-share-Analyse sind die gruppenspezifischen Einkommensmittelwerte (und damit auch der jeweilige gesamtgesellschaftliche Äquivalenzeinkommensmittelwert) auf die Niveaus des Basisjahres 1995 fixiert worden (Übersicht 1).

Diese Shift-share-Analyse abstrahiert also von Veränderungen im querschnittlichen Alters-Einkommens-Profil über die Zeit hinweg. Anders ausgedrückt: Der komparativ-statische Vergleich dieser fiktiven Ungleichheitswerte mit den jeweils faktisch gegebenen Ungleichheitswerten ermöglicht (indirekt) Einblicke in die Ungleichheitswirkungen von Determinanten der Alters-Einkommens-Profile wie altersabhängige Produktivitätsentwicklungen oder Senioritätsentlohnung.

In Tabelle 1, Spalte $c$ sind die entsprechenden Berechnungsergebnisse dargelegt. Es zeigt sich, dass wegen der im Untersuchungszeitraum doch recht geringen Unterschiede in den gruppenspezifischen Mittelwerten die Ungleichheitswerte der faktischen und kontrafaktischen Situation relativ nahe beieinander liegen. Hierbei sind bis 2000 die kontrafaktischen CV-Werte (leicht) höher als die faktischen CV-Werte gewesen; von 2001 bis 2007 galt das Umgekehrte.

Grundsätzlich war die relative Einkommenslage der mittleren Generation - im Durchschnitt und im Zeitablauf (schwach) besser als die der beiden anderen Generationen. Ferner hat sich im
Verhältnis zwischen der jüngsten und der ältesten Generation über die Zeit hinweg - in Durchschnittsperspektive - ein Bedeutungswandel ergeben. Seit der deutschen Einigung ist der Prozentwert aus gruppenspezifischem und gesamtgesellschaftlichem Äquivalenzeinkommens-Mittelwert bei der ältesten Generation - d.h. deren relative Einkommensposition - nahezu ständig gestiegen. Demgegenüber ist die relative Einkommensposition der jüngsten Generation seitdem fast beständig gefallen (vgl. Faik 2008, S. 426ff.). Als Gründe für diese zunehmenden relativen Verbesserungen der ältesten Generation kommen im Beobachtungszeitraum von 1995 bis 2007 nicht zuletzt (Kohorten-)Effekte wie gestiegene Vermögensbestände und damit korrespondierende höhere Vermögenseinkommen, relativ hohe Rentenanpassungen im Zeitablauf usw. aufseiten der Älteren infrage.

\section{KONSTANTE GRUPPENINTERNE UNGLEICHHEIT}

In einer weiteren Shift-share-Analyse wird nachfolgend die gruppeninterne Einkommensungleichheit $\mathrm{CV}_{\mathrm{g}}$ des Jahres 1995 über den Beobachtungszeitraum 1995-2007 hinweg konstant gehalten (Übersicht 1).

Empirisch offenbart sich in diesem $\mathrm{Zu}$ sammenhang die herausragende Rolle der Intragruppen-Ungleichheit bei der Bestimmung der gesamten Einkommensungleichheit. Dies wird anhand von Tabelle 1, Spalted daran deutlich, dass sich bei Konstanz der gruppeninternen Ungleichheitswerte (auf dem Niveau des Jahres 1995) die Gesamtungleichheit der Haushaltsnettoäquivalenz- 
einkommen zwischen 1995 und 2007 kaum verändert hätte - im Unterschied zum steigenden Trend der faktischen Einkommensungleichheit in Deutschland vor allem seit der Jahrtausendwende. Es hätten sich entsprechend seit 2001 bei konstanter gruppeninterner $\mathrm{CV}$-Ungleichheit deutlich geringere Gesamt-CV-Ungleichheitswerte ergeben (im Vergleich zu den jeweiligen faktischen Ungleichheitswerten), 2007 zum Beispiel in einem Ausmaß von immerhin fast $61 \%$. Dieser Befund ist die Folge einer - im Hinblick auf das Verteilungsmerkmal Haushaltsnettoäquivalenzeinkommen-im Zeitablauf (tendenziell) gestiegenen Heterogenität der drei betrachteten Generationen.

Es haben sich seit der Jahrtausendwende für sämtliche betrachteten Altersgruppen-zumindest von der Tendenz her - gruppeninterne Ungleichheitsanstiege ergeben. Von 1999 an war die Ungleichheit in der ältesten Generation (in der Regel) jeweils am höchsten und die in der jüngsten Generation jeweils am niedrigsten (siehe etwa Faik 2008, S. 425f.).

Als Gründe für die beobachteten Anstiege in der jeweiligen gruppeninternen Ungleichheit der Haushaltsnettoäquivalenzeinkommen seit der Jahrtausendwende kommen unter anderem infrage:

- Die im Vergleich zu den Arbeitseinkommen gestiegene Bedeutung der Kapitaleinkommen, welche typischerweise ungleichmäßiger als die Arbeitseinkommen, aber auch als die Transfereinkommen, verteilt sind (Grömling 2006, S. 38; Frick/Grabka 2010),

- die Auswirkungen des sozialpolitischen Paradigmenwechsels, wie er in der „Agenda 2010 “ dokumentiert worden ist, und hierbei nicht zuletzt die zunehmende Bedeutung von Nichtnormarbeitsverhältnissen mit der Folge einer wachsenden Spreizung der Arbeitseinkommen (Eichhorst/ Marx 2009) sowie

- haushaltsstrukturelle Veränderungen (etwa in Form wachsender Bildungs- und Einkommenshomogamie bei der Haushaltsformation; vgl. Hradil 2005, S. 444; Jenkins 1995; Martin 2006; OECD 2008 oder Peichl et al. 2010).

\subsection{PROJEKTIONEN DER EINKOM- MENSUNGLEICHHEIT IN DEUTSCHLAND BIS 2015}

Angesichts des Alterungsprozesses der bundesdeutschen Gesellschaft interes- siert auch die künftige Entwicklung der Einkommensungleichheit in Deutschland. Dieser Frage soll im Folgenden mit Hilfe von Projektionen auf der Grundlage verschiedener Szenarien nachgegangen werden. Da die nachfolgenden Betrachtungen auf Trendfunktionen für die ökonomischen Variablen mit der Implikation von Strukturkonstanz bezüglich u. a. der ökonomischen und gesellschaftlichen Bedingungen fußen, sollte der Projektionszeitraum nicht allzu weit in der $\mathrm{Zu}$ kunft liegen. Daher wird im Folgenden der Projektions-Endzeitpunkt auf das Jahr 2015 gelegt. Wegen zum Teil nicht signifikanter Parameterwerte in den diversen Trendfunktionen sind die nachstehenden Projektionen unbedingt vorsichtig zu interpretieren.

Im Einzelnen werden folgende $\mathrm{Zu}$ kunftsszenarien voneinander unterschieden:

(1) In Szenario I werden die Bevölkerungsdaten der 12. koordinierten Bevölkerungsvorausschätzung des Statistischen Bundesamtes für die drei voneinander unterschiedenen Altersgruppen zugrunde gelegt. Es wird auf die mittlere Bevölkerungsvorausschätzung mit einer konservativen Zuwanderungsvariante Bezug genommen. Da die alternative Verwendung einer höheren Zuwanderung im Rahmen der mittleren Bevölkerungsprojektion - eigenen Berechnungen zufolge lediglich marginale, vernachlässigenswerte Ungleichheitsunterschiede im Vergleich zur zuerst genannten Variante zur Folge hatte, erscheint die Bezugnahme auf nur eine der beiden zur Auswahl stehenden (Zuwanderungs-)Varianten gerechtfertigt. Die (Äquivalenz-)Einkommensmittelwerte und -streuungen des Jahres 2007 als „aktueller Rand“ werden konstant gehalten, sodass es sich bei Szenario I um eine rein demografische Shift-share-Projektion bis zum Jahr 2015 handelt.

(2) In Szenario II wird ergänzend zu Szenario I unterstellt, dass sich die zwischen 1995 und 2007 beobachteten Trends bezüglich der (Äquivalenz-)Einkommensmittelwerte in den drei betrachteten Altersgruppen bis zum Ende des Projektionszeitraums (2015) ungebrochen fortsetzen werden. ${ }^{8}$ Als Nebenbedingung wird berücksichtigt, dass die mit den jeweiligen Bevölkerungsanteilen gewichteten gruppenbezogenen Einkommensanteile auf der Gesamtebene
1,0 ergeben müssen. Die gruppeninternen Ungleichheitswerte werden jeweils konstant auf dem Niveau des Jahres 2007 gehalten.

(3) In Szenario III werden die Szenarien I und II um die zwischen 1995 und 2007 beobachteten Trends bezüglich der gruppeninternen (Äquivalenz-) Einkommensungleichheit in den drei Altersklassen ergänzt. ${ }^{9}$ Dieses Szenario beinhaltet demnach die Kombination aus den angenommenen Veränderungen bezüglich Bevölkerungs- und (Äquivalenz-)Einkommensanteilen der drei Altersgruppen und in Bezug auf die gruppeninternen (Äquivalenz-)Einkommensungleichheiten. Es handelt sich um eine totalanalytische Betrachtung der direkten und indirekten Demografieeinflüsse auf die gesamte Einkommensungleichheit.

Abbildung 2 gibt Auskunft über die projizierten Gesamtungleichheitswerte für die einzelnen Szenarien. An dieser Stelle ist explizit nochmals darauf hinzuweisen, dass die indirekten Demografieeffekte ausschließlich zeitreihenanalytisch über Trends operationalisiert wurden und nicht etwa das Ergebnis eines elaborierten ökonomischen Modells waren; es handelt sich demgemäß sozusagen um eine reduzierte Modellstruktur.

Abbildung 2 zeigt für Szenario I im Projektionszeitraum lediglich geringfügige Ungleichheitsänderungen; es wird allenfalls ein mäßiger Ungleichheitsanstieg

8 Folgende Trendfunktionen sind - jeweils im Sinne bester Anpassungen an die tatsächlichen Daten - geschätzt worden: Bis 29 Jahre: $v=0,0002$ $\mathrm{t}^{2}-0,005^{*} \mathrm{t}+0,919 * * *\left(\mathrm{R}^{2}=0,667\right), 30-59$ Jahre: $v=-0,0003 t^{2}-0,004 t+1,113^{* * *}\left(R^{2}\right.$ $=0,398), 60+$ Jahre: $-0,001 * * * \mathrm{t}^{2}+0,014 * * * \mathrm{t}$ $+0,910^{* * *}\left(R^{2}=0,841\right)$; mit: $v=$ Einkommensanteil, $\mathrm{t}=$ Zeit-Variable (mit $\mathrm{t}=0$ für 1995), $\mathrm{R}^{2}=$ korrigiertes Bestimmtheitsmaß, ***: signifikant auf dem 1-\%-Niveau der Irrtumswahrscheinlichkeit, ${ }^{* *}$ : signifikant auf dem 5-\%-Niveau der Irrtumswahrscheinlichkeit, * : signifikant auf dem 10-\% -Niveau der Irrtumswahrscheinlichkeit.

9 In diesem Kontext wurden folgende Trendfunktionen geschätzt: Bis 29 Jahre: $C V=0,001 \mathrm{t}^{2}-0,010$ $t+0,141^{* *}\left(R^{2}=0,699\right), 30-59$ Jahre: $C V=$ $0,003 t^{2}-0,013 t+0,167 * * *\left(R^{2}=0,793\right), 60+$ Jahre: $0,113^{* * *} \mathrm{e}^{0,135^{* * *} \mathrm{t}}\left(\mathrm{R}^{2}=0,789\right)$; mit: $\mathrm{CV}=$ normierter Variationskoeffizient, $\mathrm{t}=$ Zeit-Variable (mit $\mathrm{t}=0$ für 1995), $\mathrm{R}^{2}=$ korrigiertes Bestimmtheitsmaß, ***: signifikant auf dem 1-\%-Niveau der Irrtumswahrscheinlichkeit, ${ }^{* *}$ : signifikant auf dem 5-\%-Niveau der Irrtumswahrscheinlichkeit, *: signifikant auf dem 10-\%-Niveau der Irrtumswahrscheinlichkeit. 
Abb. 2: Projektionen der Einkommensungleichheit in

Deutschland 2010-2015*

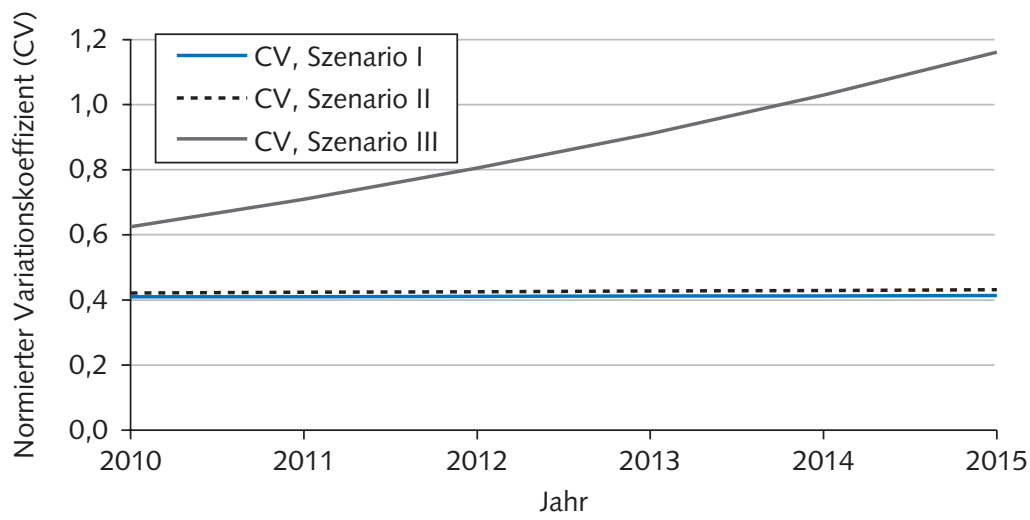

*personelle Haushaltsnettoäquivalenzeinkommen, normierte Variationskoeffizienten, Projektionsbezugjahr: 2007

bis 2015 sichtbar. Dieses Ergebnis bestätigt den bekannten Befund vergleichsweise geringer direkter Demografieeffekte auf die personelle Einkommensungleichheit (vgl. Burtless 2009, S. 445f.).

Nur geringfügig stärker fällt der Ungleichheitsanstieg im Projektionszeitraum in Szenario II aus. Für diesen mäßigen Ungleichheitsanstieg spielen die projizierten Bedeutungsgewinne der ältesten Bevölkerungsgruppe bezüglich der jeweiligen (Äquivalenz-)Einkommensanteile insofern die entscheidende Rolle, als dies die Gewichtung der Gruppe mit der im Projektionsbezugsjahr 2007 größten internen Einkommensungleichheit erhöht.

Aus Szenario III folgt - wie Abbildung 2 ausweist - ein gegenüber den Szenarien I und II wesentlich markanterer Ungleichheitsanstieg, welcher aus der in die Zukunft projizierten (weiteren) Erhöhung der seit der Jahrtausendwende beobachteten Heterogenität der (Äquivalenz-)Einkommensverhältnisse innerhalb der einzelnen Altersgruppen resultiert. Dies verweist darauf, dass auch andere als rein demografische Effekte für die (künftige) Ungleichheit der Haushaltsnettoäquivalenzeinkommen bedeutsam sind.

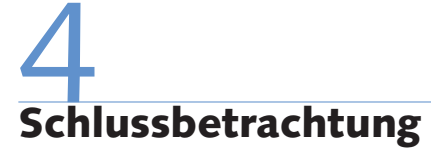

Auf die personelle Einkommensungleichheit wirken demografische Einflüsse direkt und indirekt ein. Hierbei kommen auf der indirekten Einflussebene als Übertragungskanäle der Arbeitsmarkt, der Kapitalmarkt, das Steuer-Transfer-System, die Einkommensverwendung und die makroökonomische Ebene in Betracht. Die in diesem Beitrag behandelten Berechnungsergebnisse offenbarten einen eher mäßigen direkten Einfluss der Demografie auf die gemessene Einkommensungleichheit (im Querschnitt). Entscheidender sind die indirekten Demografieeffekte, und hier wiederum vor allem Entwicklungen der gruppeninternen Ungleichheiten, die - unseren Projektionen zufolge - für die Zukunft eine (wesentlich) ungleichmäßigere personelle Einkommensverteilung in Deutschland als bis dato erwarten lassen. Dies könnte unter anderem die Folge eines zunehmenden Bedeutungsgewinns der ungleichmäßiger verteilten Kapitaleinkommen gegenüber den Arbeitseinkommen, aber eventuell auch das Ergebnis einer zunehmenden Bildungs- und Einkommenshomogamie beim Heiratsverhalten beziehungsweise bei der Haushaltsbildung sein. Hierzu stehen umfassendere wissenschaftliche Untersuchungen noch aus, mit deren Hilfe die vorstehenden Vermutungen fundiert beziehungsweise widerlegt werden könnten.

Über die Querschnittsanalysen dieses Beitrags hinaus erlaubt allerdings erst die Berücksichtigung der Längsschnittdimension die Trennung zwischen reinen Alters- und Kohorteneffekten. Hierdurch - sowie gegebenenfalls auch mit einer über den Wohlstandsindikator Einkommen hinausgehenden, d.h. mehrdimensionalen Wohlstandsanalyse unter Berücksichtigung beispielsweise des Haushaltsvermögens - kann der Frage nach der Generationengerechtigkeit (noch) umfassender nachgegangen werden als bei einer ausschließlichen Querschnittsanalyse (beziehungsweise auch im Vergleich zu einer nur auf das Haushaltseinkommen fokussierten eindimensionalen Wohlstandsbetrachtung).

Die Projektionsbefunde dieses Beitrags fußten überdies auf Zeitreihentrends. Einer weiterführenden, komplexeren Analyse muss es vorbehalten bleiben, die indirekten Demografieeffekte auf die personelle Einkommensverteilung mittels eines ökonomischen Modells zu fundieren. In diesem Kontext bietet sich ein dynamisches allgemeines (OLG-)Gleichgewichtsmodell ${ }^{10}$ an . Im Rahmen eines solchen Modells könnte zum Beispiel eine Abflachung der AltersEinkommens-Profile modelliert werden, wie sie von Schröder (2004, S. 289) wegen der vermuteten Verteuerung der Senioritätsentlohnung, und wegen relativer Einkommenseinbußen der älteren Erwerbstätigen als Folge des Anstiegs der relativen Kohortenstärke der älteren Erwerbstätigen angenommen wird und was die oben präsentierten Ungleichheitsanstiege etwas abbremsen würde.

10 OLG steht für Overlapping Generations; siehe etwa die Ausführungen in Heer/Maussner 2009. 
Ando, A. K./Modigliani, F. (1963): The "Life-Cycle" Hypothesis of Saving: Aggregate Implications and Tests, in: American Economic Review, 53 (1), S. 55-84

Becker, G. S. (1964): Human Capital, New York

Börsch-Supan, A./Essig, L. (2002): Sparen in Deutschland. Ergebnisse der ersten SAVE-Studie, Köln

Burkhauser, R. V./Butrica, B. A./Daly, M. C./Lillard, D. R. (2001): The Cross-National Equivalent File: A product of cross-national research, in: Becker, I./Ott, N./Rolf, G. (Hrsg.): Soziale Sicherung in einer dynamischen Gesellschaft, Frankfurt am Main, S. 354-376

Burtless, G. (2009): Demographic Transformation and Economic Inequality, in: Salverda, W./Nolan, B./Smeeding, T. M. (Hrsg.): The Oxford Handbook of Economic Inequality, Oxford, S. 435-454 Eichhorst, W./Marx, P. (2009): Reforming German Labor Market Institutions: A Dual Path to Flexibility, IZA-Diskussionspapier 4100, Bonn Faik, J. (2008): Sehen die Jungen alt aus? Generative Aspekte von Wohlstand, in: Deutsche Rentenversicherung, 63 (5), S. 419-434 Faik, J. (2010a): Methodical Settings in Analyses of the Income Distribution - Some Simple Mathematical Comments, FaMa-Diskussionspapier 1, Frankfurt am Main

Faik, J. (2010b): Socio-Demography and Income Inequality - An Overview, FaMa-Diskussionspapier 4, Frankfurt am Main

Frick, J./Grabka, M. M. (2010): Accounting for Imputed and Capital Income Flows in Inequality Analyses, in: Besharov, D./Couch, K. (Hrsg.): Measuring Poverty, Income Inequality, and Social Exclusion, Oxford Grömling, M. (2006): Die Lohnquote - ein statistisches Artefakt und seine Interpretationsgrenzen, in: IW-Trends, 33 (1), S. 35-48

Hauser, R. (2007): Soziale Gerechtigkeit in Deutschland - Zieldimensionen und empirische Befunde am Beispiel der Generationengerechtigkeit, in: Empter, S./Vehrkamp, R. B. (Hrsg.): Soziale Gerechtigkeit - eine Bestandsaufnahme, Gütersloh, S. 136-167

Heer, B./Maussner, A. (2009): Dynamic General Equilibrium Modeling. Computational Methods and Applications, Heidelberg
Heerink, N. (1994): Population Growth, Income Distribution, and Economic Development. Theory, Methodology, and Empirical Results, Berlin

Hradil, S. (2005): Soziale Ungleichheit in Deutschland, Wiesbaden Jenkins, S. P. (1995): Accounting for Inequality Trends: Decomposition Analysis for the UK, 1971-86, in: Economica, 62 (245), S. 29-63 Kuznets, S. (1976): Demographic Aspects of the Size Distribution of Income: An Exploratory Essay, in: Economic Development and Cultural Change, 25 (1), S. 1-94

Martin, M. A. (2006): Family Structure and Income Inequality in Families with Children, 1976 to 2000, in: Demography, 43 (3), S. 421-445

Mincer, J. (1974): Schooling, Experience, and Earnings, New York OECD (2008): Growing Unequal? Income Distribution and Poverty in OECD Countries, Paris

Paglin, M. (1975): The Measurement and Trend of Inequality: A basic Revision, in: American Economic Review, 65 (4), S. 598-609

Peichl, A./Pestel, N./Schneider, H. (2010): Does Size Matter? The Impact of Changes in Household Structure on Income Distribution in Germany, IZA-Diskussionspapier 4770, Bonn

Pohmer, K. (1989): Der Einfluß der Bevölkerungsentwicklung auf die personelle Verteilung von Einkommen und Vermögen, in: Felderer,

B. (Hrsg.): Einkommensverteilung und Bevölkerungsentwicklung, Berlin, S. $85-119$

Schröder, Ch. (2004): Verteilung, in: Institut der deutschen Wirtschaft Köln (Hrsg.): Perspektive 2050. Ökonomik des demographischen Wandels, Köln, S. 265-291

von Weizsäcker, R. K. (1994): Demographischer Wandel, Staatshaushalt und Einkommensverteilung, in: Felderer, B. (Hrsg.): Einkommensverteilung und Bevölkerungsentwicklung, Berlin, S. 31-84

Western, B./Bloom, D./Percheski, Ch. (2008): Inequality among American Families with Children, 1975 to 2005, in: American Sociological Review, 73 (6), S. 903-920 